\title{
Involvement of Annexin A2 in Serum-MAF Dependent Phagocytic Activation of Macrophages
}

\author{
KUMPEI KAWAKATSU, MISATO MAEMURA, YOSHIKA SETA and TAKAHITO NISHIKATA
}

Frontiers of Innovative Research in Science and Technology (FIRST), Konan University, Kobe, Japan

\begin{abstract}
Background/Aim: Serum-derived macrophage activating factor (serum-MAF) is expected to have adjuvant effects through rapid phagocytic activation, which depends on F-actin accumulation in multi-layered membrane ruffles induced within 5 min after serum-MAF addition. This study aimed to elucidate the importance of annexin A2, which is a multifunctional $\mathrm{Ca}^{2+}$-binding protein related to cytoskeletal membrane dynamics, in serum-MAF signalling. Materials and Methods: Annexin A2 and F-actin localizations were analyzed via immunostaining and confocal microscopy. Using EGTA as chelator, the role of $\mathrm{Ca}^{2+}$ in serum-MAF signalling was examined. Results: Annexin A2 was found to translocate from the cytosol to the cell cortex within $30 \mathrm{~s}$ of serum-MAF stimulation. $\mathrm{Ca}^{2+}$ chelation inhibited the translocation of annexin A2, frill-like structure formation, and phagocytic activation by serum-MAF. Conclusion: Annexin $\mathrm{A} 2$ and $\mathrm{Ca}^{2+}$ were responsible for the rapid phagocytic activation by serum-MAF. This study provides an understanding of phagocytic activation in macrophages, which could be beneficial for cancer immunotherapy.
\end{abstract}

Macrophages play a pivotal role in activating the entire immune system by phagocytosing pathogens (1). Thus, the enhancement of macrophage phagocytic activity is expected to maintain human homeostasis, including cancer immunity $(1,2)$. We previously described the rapid and strong phagocytic activity of the serum derived macrophage activating factor, serum-MAF (3). Serum-MAF is a human serum treated with galactosidase and sialidase (4). Serum-

This article is freely accessible online.

Correspondence to: Takahito Nishikata, Frontiers of Innovative Research in Science and Technology (FIRST), Konan University, 71-20, Minatojima-minamimachi, Chuo-ku, Kobe 650-0047, Japan. Tel: +81 783031349, Fax: +81 783031495, e-mail: nisikata@konan-u.ac.jp

Key Words: THP-1, serum-MAF (serum-derived macrophage activating factor), annexin $\mathrm{A} 2, \mathrm{~F}$-actin dynamics, $\mathrm{Ca}^{2+}$.
MAF-activated macrophages form a frill-like structure: Factin accumulates multi-layered membrane ruffles and is responsible for effective phagocytosis within $5 \min (5,6)$. The mechanisms underlying such rapid actin reorganization are intriguing at the cytological and immunological levels.

Annexin A2 is one of the actin-binding proteins. It is a multifunctional $\mathrm{Ca}^{2+}$ - and lipid-binding protein expressed in approximately all human tissues and cell types. The functions of annexin A2 include cytoskeleton-membrane interactions, and participation in a broad range of intracellular processes, such as membrane domain organization, membrane fusion, and vesicle aggregation, which are involved in many cellular functions, such as exocytosis, endocytosis, and phagocytosis (7-11).

We hypothesized that the regulation of cytoskeletonmembrane interactions is involved in rapid F-actin reorganization in frill-like structures. This study analyzed the role of endogenous annexin A2 in serum-MAF-activated macrophages.

\section{Materials and Methods}

Cell culture. The THP-1 cell line was obtained from RIKEN BRC (Tokyo, Japan). THP-1 cells were differentiated into macrophages by incubation with $400 \mathrm{ng} / \mathrm{ml} \mathrm{12-O-tetradecanoyl-13-acetate} \mathrm{(TPA;}$ Sigma-Aldrich, St. Louis, MO, USA) for $24 \mathrm{~h}$.

Immunofluorescence and confocal microscopy. Differentiated macrophages were fixed with $1.6 \%$ formaldehyde (Polysciences, Warrington, PA, USA) and stained with anti-annexin A2 antibody (1:100; GeneTex, Irvine, CA, USA) and Alexa Fluor plus 555conjugated secondary antibody (1:1000; Invitrogen, Oslo, Norway). Cells were counterstained with $0.1 \mathrm{U} / \mathrm{ml}$ Alexa Fluor 488-conjugated phalloidin (Invitrogen) and $10 \mu \mathrm{g} / \mathrm{ml}$ 4',6-Diamidino-2-phenylindole dihydrochloride (DAPI; Nacalai Tesque, Kyoto, Japan). Cells were observed under an A1RHD25 confocal microscope (Nikon, Tokyo, Japan).

Phagocytic assay. Phagocytosis assays were performed as described previously (3). Cells were cultured in 96-well plates and treated with $8 \mu \mathrm{g} / \mathrm{ml}$ serum MAF for $5 \mathrm{~min}$, followed by washing with $1 \times \mathrm{PBS}$. 10, 30, and $60 \mathrm{~min}$ after adding $6 \mu \mathrm{g} /$ well $\mathrm{pH}$-sensitive beads, phagocytosed and internalized beads were measured using a 


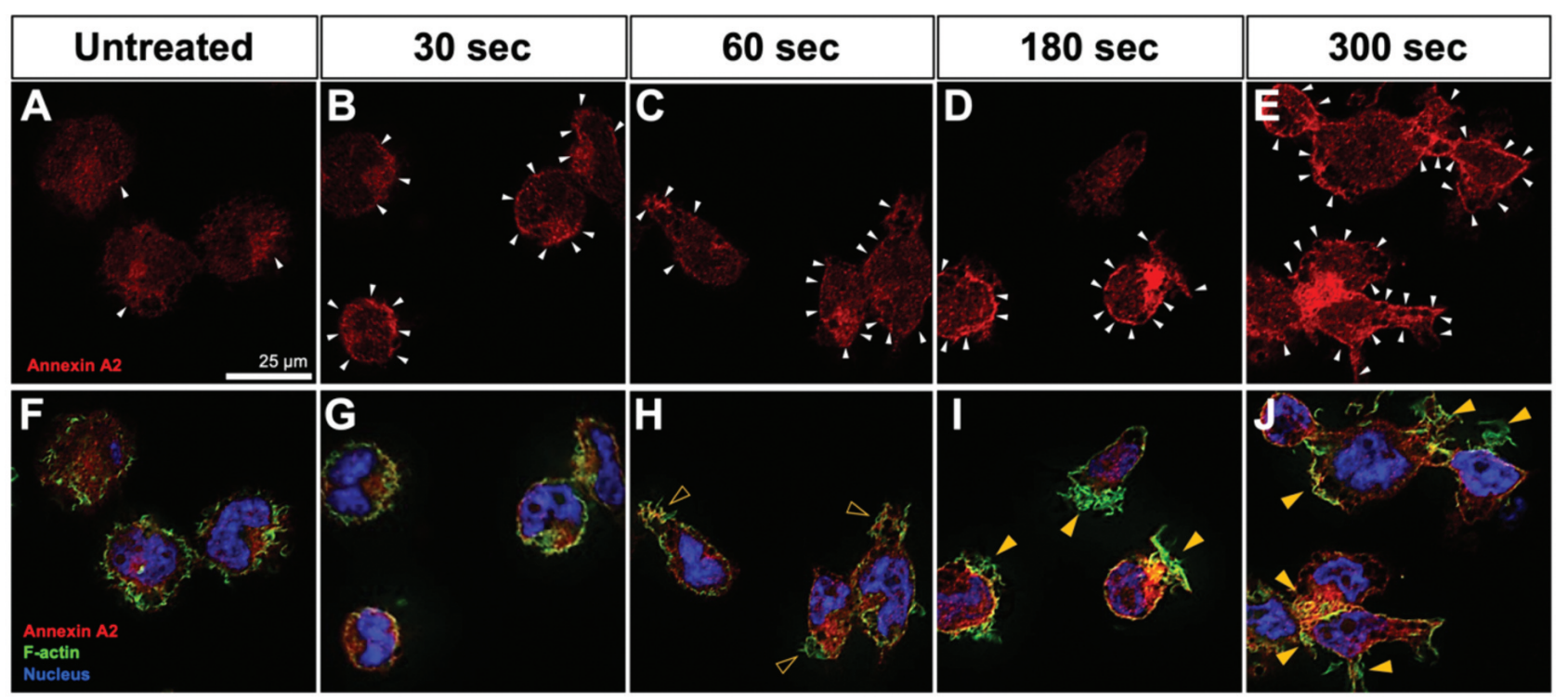

Figure 1. The changes in the localization of annexin A2 and F-actin. Macrophages were fixed at $0 \mathrm{~s}(A, F$; Untreated), $30 \mathrm{~s}(B, G), 60 \mathrm{~s}(\mathrm{C}, \mathrm{H})$, $180 \mathrm{~s}(D, I)$, and $300 \mathrm{~s}(E, J)$ after addition of $8 \mu \mathrm{g} / \mathrm{ml}$ serum macrophage activating factor (MAF) and stained for annexin A2 (red), F-actin (green), and nuclei (blue). Upper images (A-E) show only annexin A2 staining, and lower images (F-J) show merged images. White arrowheads indicate cortical annexin A2 staining. Blank and filled yellow arrowheads indicate the buds and fully formed frill-like structures, respectively. Note that total annexin A2 staining was increased from 180 s concomitant with frill-like structure formation; however, most of the annexin A2 disappeared from the vigorously ruffling membrane. Scale bars; $25 \mu \mathrm{m}$.
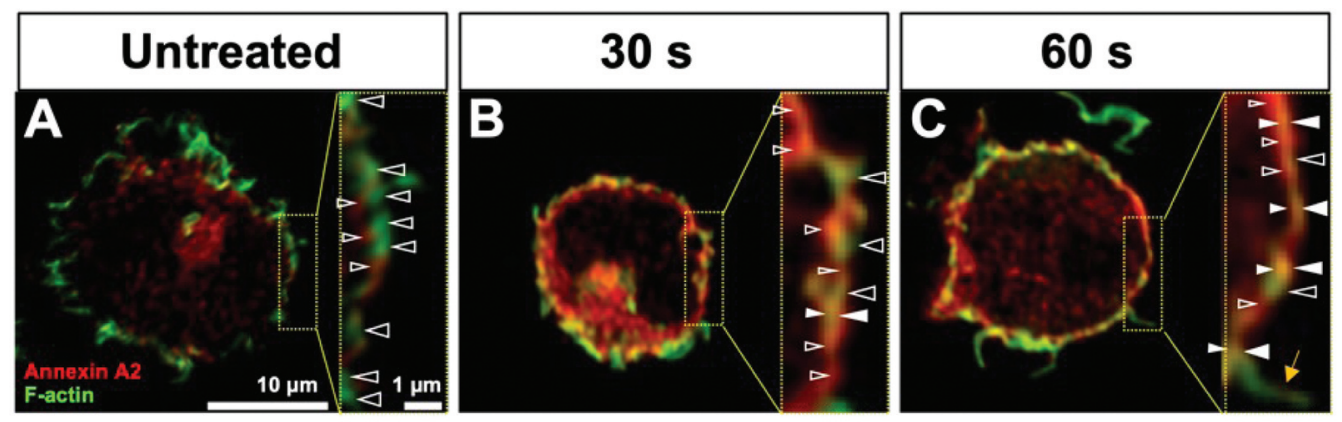

Figure 2. The changes in the localization of annexin A2 and F-actin just after addition of serum-derived macrophage activating factor (MAF). These are enlarged images of cells from the same experiment as in Figure 1, without DAPI staining. A higher magnification of each inserted dotted square is indicated on the left side. Large and small arrowheads indicate the cortical localization of F-actin and annexin A2, respectively. Blank and filled arrowheads indicate areas of localisation of only F-actin or annexin A2 or areas where they are colocalized, respectively. The yellow arrow indicates small-membrane ruffling. Cortical colocalization of annexin A2 and F-actin gradually increased up to $60 \mathrm{~s}$. Scale bars: $10 \mu \mathrm{m}$ and $1 \mu \mathrm{m}$ as indicated.

fluorescence plate reader. $\mathrm{pH}$-sensitive beads were AcidiFluor ORANGE-NHS (Goryo Chemical, Hokkaido, Japan)-labelled magnetic beads (Dynabeads ${ }^{\circledR}$ Protein G; Invitrogen). The phagocytic activity of macrophages was evaluated as the internalized bead ratio measured by fluorescence (IBRf), which was calculated using the following formula:

$\operatorname{IBRf}(\%)=($ fluorescence intensity of internalized beads $) /$ (fluorescence intensity of all beads) $\times 100$.

\section{Results}

Serum-MAF induces translocation of annexin A2 to the cell cortex. As shown in Figure 1, in untreated macrophages (without serum-MAF), annexin A2 was observed as tiny dots scattered within the cytoplasm (Figure 1A, F). Within $30 \mathrm{~s}$ after serum-MAF treatment (Figure 1B, G), annexin A2 accumulated in the cortical region. At $60 \mathrm{~s}$ after serum-MAF 


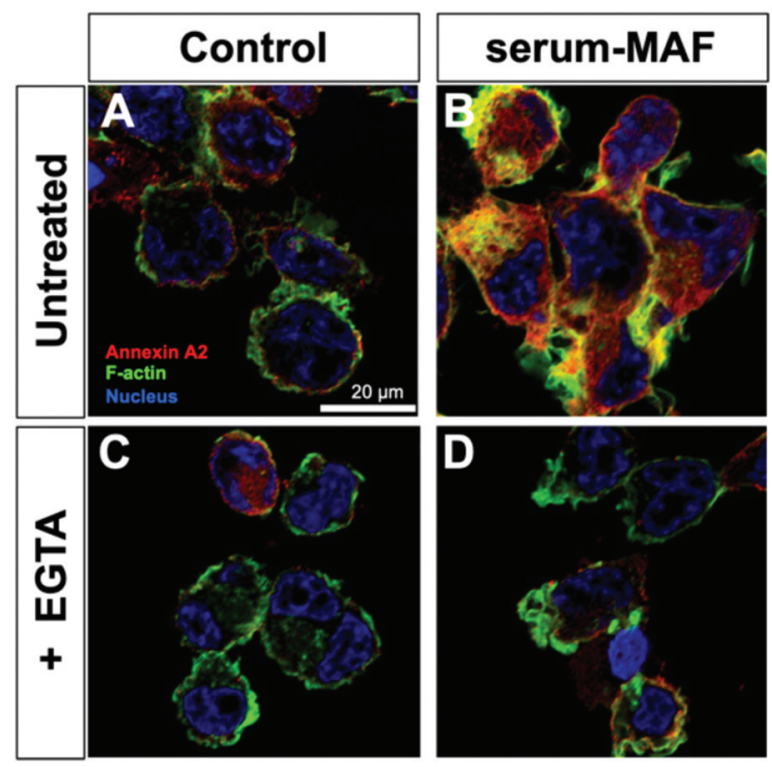

Figure 3. Effect of $\mathrm{Ca}^{2+}$ chelation on annexin $\mathrm{A} 2$ and F-actin localization. Macrophages preincubated with $(C, D)$ or without $(A, B)$ EGTA $(2.5 \mathrm{mM})$ for $60 \mathrm{~min}$ were treated with $(B, D)$ or without $(A, C)$ $8 \mu \mathrm{g} / \mathrm{ml}$ serum-MAF for $5 \mathrm{~min}$. Cells were stained for annexin A2 (red), F-actin (green), and nuclei (blue). Scale bar; $20 \mu \mathrm{m}$.

treatment, thin actin accumulation at the edge of membrane ruffling started to be observed as a bud for a newly formed frill-like structure (Figure 1H). In some ruffles, annexin A2 cortical accumulation was observed around the basal region of the ruffles (Figure 1C). After 180 and $300 \mathrm{~s}$, an evident frill-like structure was formed (Figure 1I and J). Although the total fluorescent intensity of annexin A2 was increased, particularly in some cortical regions, annexin A2 was no longer present in the fully formed frill-like structure (Figure $1 \mathrm{D}$ and $\mathrm{E})$.

Relationship between F-actin and annexin-A2 accumulation in the cortical region. In the absence of serum-MAF, only a few small dots of annexin A2 were also observed in the cortex (Figure 2A, small blank arrowheads). The distribution of annexin A2 and F-actin was mutually exclusive. When F-actin resided in the cortex, annexin A2 dots were situated beneath the membrane (Figure 2A). Furthermore, 30 or $60 \mathrm{~s}$ after serum-MAF addition, cortical accumulations of annexin $\mathrm{A} 2$ became evident and lined up in some cortical areas (Figure $2 \mathrm{~B}$ and $\mathrm{C}$ ). F-actin and annexin A2 still showed mutually exclusive localization in some cortical regions (blank arrowheads), although their colocalization was obvious (filled arrowheads). Although annexin A2 accumulation beneath cortical F-actin (small arrowheads) was still observed at $30 \mathrm{~s}$, these two became aligned in the cortical region at $60 \mathrm{~s}$. Annexin A2 was

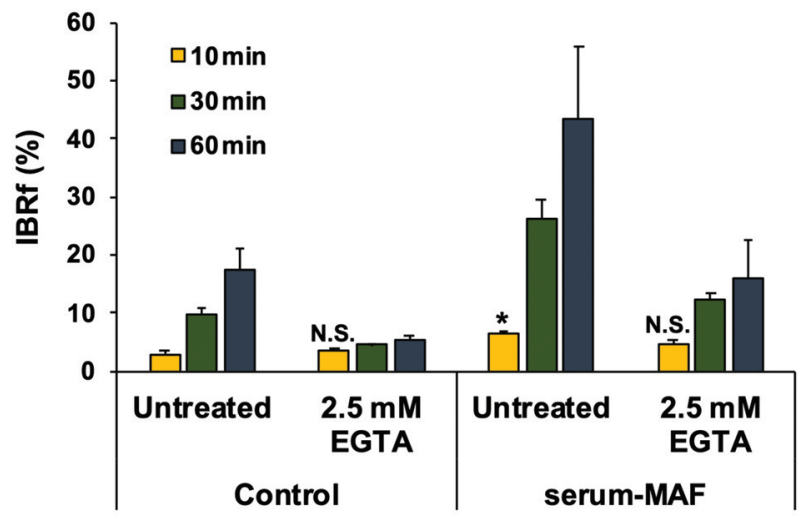

Figure 4. Effect of $\mathrm{Ca}^{2+}$ chelation on phagocytic activity. Macrophages were preincubated with or without EGTA $(2.5 \mathrm{mM})$ for $60 \mathrm{~min}$. The phagocytic activities of macrophages treated with (serum-MAF) or without (control) $8 \mu \mathrm{g} / \mathrm{ml}$ serum-MAF for 5 min were measured. Error bars represent standard deviation. $* p<0.05$, compared to the untreated control at $10 \mathrm{~min}$ (t-test; $n=3)$. N.S.: Not significant.

excluded from the flattened membrane ruffling (Figure 2C, yellow arrow).

$\mathrm{Ca}^{2+}$ chelator inhibited annexin A2 accumulation and frilllike structure formation. Intracellular $\mathrm{Ca}^{2+}$ is considered to mediate annexin A2-activation (12). When EGTA (2.5 mM) was added to the culture medium, cortical annexin A2 accumulation within $60 \mathrm{~s}$ (data not shown) and increased annexin A2 expression at 5 min (Figure 3) were inhibited. Frill-like structure formation was also suppressed by $\mathrm{Ca}^{2+}$ chelation.

$\mathrm{Ca}^{2+}$ chelation down-regulates phagocytic activity. The phagocytic activity of serum-MAF-treated macrophages showed a rapid and significant increase $(10 \mathrm{~min}$, in this experiment), in addition to the later activation (30, and 60 min; Figure 4) (3). Under $\mathrm{Ca}^{2+}$-chelated conditions (addition of EGTA (2.5 mM)), the phagocytic activity of serum-MAFtreated macrophages was down-regulated at all time points, as compared to that without EGTA. It was noted that even in $\mathrm{Ca}^{2+}$-chelated conditions, serum-MAF up-regulated phagocytic activity similar to that observed under normal conditions.

\section{Discussion}

This study concludes that annexin $\mathrm{A} 2$ and $\mathrm{Ca}^{2+}$ play an important role in the formation of frill-like structures and are thus responsible for the rapid and strong phagocytic activation by serum-MAF. The increased expression of annexin A2 from 3 min after activation corresponded to 
F-actin accumulation in the frill-like structure. Rapid (within $30 \mathrm{~s})$ translocation of annexin A2 to the cortical region was the fastest event observed to date. The binding ability of annexin A2 to both actin and membrane lipids strongly suggests that it could directly mediate the serum-MAF induced signalling upstream of actin reorganization (10). Moreover, the mutually exclusive localization pattern of Factin and annexin A2 indicated that the lifespan of annexin A2 dots in the cortex was very short due to the rapid reorganization of F-actin in the flexible frill-like structure (5).

In the EGTA experiment, it was difficult to elucidate the role of $\mathrm{Ca}^{2+}$ in the serum MAF signaling pathway. For example, $\mathrm{Ca}^{2+}$ chelation with EGTA could not distinguish between the roles of extracellular and intracellular $\mathrm{Ca}^{2+}$. However, even in the presence of EDTA, serum-MAF somewhat enhanced the phagocytic activity, suggesting the existence of a $\mathrm{Ca}^{2+}$-independent pathway. Thus, the novel serum-MAF-dependent macrophage-activating pathway should be intricate.

Therefore, the serum-MAF-dependent macrophage activating pathway is completely different from the known activation pathway triggered by some receptors, such as TLRs, FcRs, and TNFRs $(1,2)$. The annexin $\mathrm{A} 2$ and $\mathrm{Ca}^{2+}$-dependent pathway could be this novel activating pathway, particularly for the rapid response. Understanding this mechanism may help develop novel adjuvants to enhance innate and adaptive immunity and novel anticancer immunotherapy.

\section{Conflicts of Interest}

The Authors declare that no conflicts of interest exist regarding this study.

\section{Authors' Contributions}

K. K. carried out most of the experiments and wrote the initial draft of the manuscript. M. M. and Y. S. contributed to data collection and interpretation. K. K. and T. N. designed the study. T. N. conceived and coordinated the study and helped draft the manuscript. All Authors read and approved the final manuscript.

\section{Acknowledgements}

The Authors thank Dr. T. Inui (Inui Immunotherapy Clinic, Osaka, Japan) and Dr. Y. Uto (Tokushima University Graduate School, Tokushima, Japan) for providing the serum MAF. Also, the Authors would like to thank Editage (www.editage.com) for English language editing.

\section{References}

1 Gordon S: Phagocytosis: An immunobiologic process. Immunity 44(3): 463-475, 2016. PMID: 26982354. DOI: 10.1016/j.immuni. 2016.02 .026

2 Zhang R, Kobayashi Y, Kazumura K, Tsuchiya H, Morishita N, Inagawa $\mathrm{H}$ and Soma $\mathrm{G}$ : Development of an evaluation device for phagocytic activity of new phagocytes using simple and $\mathrm{pH}$ sensitive particles that do not require pre-treatment. Anticancer Res 36(7): 3613-3618, 2016. PMID: 27354631.

3 Ishikawa M, Mashiba R, Kawakatsu K, Tran NK and Nishikata T: A high-throughput quantitative assay system for macrophage phagocytic activity. Macrophage 5: e1627, 2018. DOI: 10.14800/ Macrophage. 1627

4 Yamamoto N, Naraparaju VR and Asbell SO: Deglycosylation of serum vitamin D3-binding protein leads to immunosuppression in cancer patients. Cancer Res 56(12): 2827-2831, 1996. PMID: 8665521.

5 Kawakatsu K, Ishikawa M, Mashiba R, Tran NK, Akamatsu M and Nishikata T: Characteristic morphological changes and rapid actin accumulation in serum-MAF-treated macrophages. Anticancer Res 39(8): 4533-4537, 2019. PMID: 31366556. DOI: 10.21873/anticanres. 13630

6 Mashiba R, Ishikawa M, Sumiya YU, Kawakatsu K, Tran NK and Nishikata T: Phagocytic activation of macrophages with serum MAF depends on engulfment efficiency and not migratory activity. Anticancer Res 38(7): 4295-4298, 2018. PMID: 29970564. DOI: 10.21873 /anticanres.12727

7 dos Remedios CG, Chhabra D, Kekic M, Dedova IV, Tsubakihara $\mathrm{M}$, Berry DA and Nosworthy NJ: Actin binding proteins: regulation of cytoskeletal microfilaments. Physiol Rev 83(2): 433473, 2003. PMID: 12663865. DOI: 10.1152/physrev.00026.2002

8 Hayes MJ, Rescher U, Gerke V and Moss SE: Annexin-actin interactions. Traffic 5(8): 571-576, 2004. PMID: 15260827. DOI: $10.1111 / \mathrm{j} .1600-0854.2004 .00210 . \mathrm{x}$

9 Christensen MV, Høgdall CK, Jochumsen KM and Høgdall EVS: Annexin A2 and cancer: A systematic review. Int J Oncol 52(1): 5-18, 2018. PMID: 29115416. DOI: 10.3892/ijo.2017.4197

10 Gerke V and Moss SE: Annexins: from structure to function. Physiol Rev 82(2): 331-371, 2002. PMID: 11917092. DOI: 10.1152/physrev.00030.2001

11 Patel DM, Ahmad SF, Weiss DG, Gerke V and Kuznetsov SA: Annexin A1 is a new functional linker between actin filaments and phagosomes during phagocytosis. J Cell Sci 124(Pt 4): 578588, 2011. PMID: 21245195. DOI: $10.1242 /$ jcs.076208

12 Glenney JR Jr: Calpactins: calcium-regulated membrane-skeletal proteins. Bioessays 7(4): 173-175, 1987. PMID: 2961326. DOI: 10.1002/bies.950070408

Received May 22, 2021

Revised June 4, 2021

Accepted June 5, 2021 